\title{
A CONSTRUCTION OF SIMPLE PRINCIPAL RIGHT IDEAL DOMAINS
}

\author{
P. M. COHN
}

\begin{abstract}
It is shown how simple principal ideal domains can be obtained from any principal right ideal domain by localization. When no localization is needed one can, under favourable conditions, obtain a simple principal right (but not left) ideal domain, and an easy example is given.
\end{abstract}

In their recent Cambridge tract [4] Cozzens and Faith describe a number of simple Noetherian domains and, in particular, give a sufficient condition for a skew polynomial ring to be simple, taken from [3]. But it is not hard to see that any skew polynomial ring is either itself simple or it leads to a simple (left and right) principal ideal domain by localization, provided that (essentially) no power of the defining endomorphism is inner. This generalization of Cozzen's result follows rather easily from an analysis of the ideal structure proved in Theorem 4. We also give a simple example where no localization is necessary; this leads to a simple principal right, but not left, ideal domain.

I am indebted to the referee for drawing my attention to some errors in an earlier version.

1. We begin by recalling some well-known facts, mainly to fix the terminology. Let $R$ be a principal right ideal domain; each two-sided ideal of $R$ is of the form $c R$, where $R c \subseteq c R$, i.e. $c$ is right invariant. The set $I$ of all right invariant elements $(\neq 0)$ is easily seen to be a right denominator set (cf. [2, p. 21]) and the localization $R_{I}$ is simple, for every ideal of $R_{I}$ is of the form $\mathfrak{A} R_{I}$, where $\mathfrak{A}$ is an ideal of $R$, but the generator of $\mathfrak{A}(\neq 0)$ becomes a unit in $R_{I}$, whence $\mathfrak{A} R_{I}=R_{I}$. Clearly the right ideals of $R_{I}$ are again principal, so we may state the result as

THEOREM 1. Let $R$ be a principal right ideal domain and $I$ the set of its right invariant elements $(\neq 0)$; then $I$ is a right denominator set in $R$ and the localization $R_{I}$ is a simple principal right ideal domain.

When $R$ is a (left and right) principal ideal domain, it can be shown that $R_{I}$ is a skew field precisely when $R$ is not primitive.

Theorem 1 provides us with a recipe for obtaining simple principal right ideal domains. For skew polynomial rings this programme is particularly easy

Received by the editors October 27, 1976 and, in revised form, January 10, 1977.

AMS (MOS) subject classifications (1970). Primary 16A04, 16A40; Secondary 16A08, 16A72.

Key words and phrases. Principal ideal domain, simple Noetherian domain, skew polynomial ring, localization, right invariant element, formal Laurent series. 
to carry out because of the special form taken by $I$ in this case.

2. Let $K$ be a skew field with an endomorphism $\alpha$ and an $\alpha$-derivation $\delta$ and consider the skew polynomial ring

$$
R=K[t ; \alpha, \delta]
$$

with commutation rule

$$
c t=t c^{\alpha}+c^{\delta} \quad \text { for all } c \in K .
$$

It is well known that this ring is always a principal right ideal domain, and a principal left ideal domain whenever $\alpha$ is an automorphism. It follows that $R$ is a right Ore domain and has a field of fractions $K(t ; \alpha, \delta)$. This construction has been mainly considered in the noncommutative case, for if $K$ is commutative and $\alpha \neq 1$, every $\alpha$-derivation is inner (cf. [1, p. 539]).

We observe that the case when $\alpha$ is an endomorphism of $K$ can be reduced to that of an automorphism. For let $K$ be any skew field with an endomorphism $\alpha$, and write $K_{n}(n \geqslant 0)$ for the image of $\alpha^{n}$. Since $\alpha^{n}$ is clearly injective, it provides an isomorphism between $K=K_{0}$ and $K_{n}$. Now for each $m=1,2, \ldots$ take an isomorphic copy $K_{-m}$ of $K$ and embed $K_{-m}$ in $K_{-m-1}$ by identifying it with the image of $\alpha$. In this way we obtain a filtration

$$
\cdots \subseteq K_{n} \subseteq K_{n-1} \subseteq \cdots \subseteq K_{1} \subseteq K_{0} \subseteq K_{-1} \subseteq K_{-2} \subseteq \ldots,
$$

whose union is again a skew field, which will be written $K^{[\alpha]}$. Since $\alpha$ : $K_{n} \rightarrow K_{n+1}$ is defined as an isomorphism for all $n \in \mathbf{Z}, \alpha$ is an automorphism of $K^{[\alpha]}$. Another, perhaps more explicit, way of constructing $K^{[\alpha]}$ is to form the skew field of fractions $K(t ; \alpha)$ (with zero derivation). This has an inner automorphism induced by $t$ which on $K$ agrees with $\alpha$; hence $K_{-m}=t^{m} K t^{-m}$ and so $K^{[\alpha]}=\cup_{m=1}^{\infty} t^{m} K t^{-m}$. The first method of constructing $K^{[\alpha]}$ is clearly equivalent to this, and it has the advantage of emphasizing its intrinsic nature. To give an example, the centre $\mathscr{Z}\left(K^{[\alpha]}\right)$ of $K^{[\alpha]}$ consists of all $a \in \mathscr{Z}(K)$ such that $a^{\alpha^{n}} \in \mathscr{Z}(K)$ for all $n$, and the inverse images under $\alpha^{m}$ of such elements. We shall want to know the centralizer of $K$ in $K(t ; \alpha, \delta)$; it is described by

LEMMA 2. Let $K$ be a skew field with an endomorphism $\alpha$, and assume that for any $r>0, m \geqslant 0, K$ has no inner automorphism which agrees with $\alpha^{r}$ on $\operatorname{im} \alpha^{m}$. Then for any $\alpha$-derivation $\delta$ of $K$, the centralizer of $K$ in $K(t ; \alpha, \delta)$ is contained in the centralizer of $K$ in $K^{[\alpha]}$.

Proof. We have $K(t ; \alpha, \delta) \subseteq K^{[\alpha]}(t ; \alpha, \delta)$, and the latter can be embedded in the skew field of formal Laurent series in $t^{-1}$. This consists of all series $t^{r} a_{r}+t^{r-1} a_{r-1}+\ldots\left(a_{i} \in K^{[\alpha]}\right)$, with the usual multiplication determined by (2). Note that we cannot form Laurent series in $t$, because by (2) the multiplication is not continuous in the $t$-adic topology, and we need an automorphism to be able to collect negative powers of $t$ on the left of the coefficients. Now let $u=t^{r} a_{0}+t^{r-1} a_{1}+\ldots\left(a_{0} \neq 0\right)$ centralize $K$; then for any $b \in K, u b=t^{r} a_{0} b+\ldots$ and $b u=b t^{r} a_{0}+\ldots=t^{r} b^{\alpha^{r}} a_{0}+\ldots$; 
hence on equating coefficients we find

$$
b^{\alpha} a_{0}=a_{0} b \quad \text { for all } b \in K .
$$

Thus $\alpha^{r}$ is inner on $K$, induced by $a_{0} \in K_{-m}$ say; hence $\alpha^{r}$ agrees on im $\alpha^{m}$ with an inner automorphism of $K$, which contradicts the hypothesis unless $r=0$. Now (3) reduces to $b a_{0}=a_{0} b$, i.e. $a_{0}$ centralizes $K$, hence so does $u-a_{0}$. But this begins with a negative power of $t$, so by repeating the previous argument we find that $u-a_{0}=0$, and the lemma follows.

3. Let us return to the ring $R=K[t ; \alpha, \delta]$ and examine its ideals. Every right ideal of $R$ is of the form $f R$, where $f$ is a polynomial in $t$, which may without loss of generality be taken to be monic. The condition for $f$ to be right invariant (and hence for $f R$ to be two-sided) is that for each $g \in R$ there should exist $g_{1} \in R$ such that $g f=f g_{1}$; in particular, we must have

$$
c f=f c_{1} \quad \text { for all } c \in K ; \quad t f=f(t c+d) .
$$

Here $c_{1}, c, d \in K$, by a comparison of degrees. Let $f$ have degree $n$ :

$$
f=t^{n}+t^{n-1} a_{1}+\cdots+a_{n}
$$

then $c f=t^{n} c^{\alpha^{n}}+\ldots, f c_{1}=t^{n} c_{1}+\ldots$; hence by equating highest terms we find that $c_{1}=c^{\alpha^{n}}$. Similarly we have $t f=t^{n+1}+t^{n} a_{1}+\ldots$,

$$
\begin{aligned}
f(t c+d) & =t^{n+1} c+t^{n} d+t^{n-1} a_{1} t c+\cdots \\
& =t^{n+1} c+t^{n}\left(d+a_{1}^{\alpha} c\right)+\ldots ;
\end{aligned}
$$

it follows that $c=1, d+a_{1}^{\alpha}=a_{1}$. Thus we have

THEOREM 3. In any skew polynomial ring $R=K[t ; \alpha, \delta]$ with endomorphism $\alpha$ and $\alpha$-derivation $\delta$, the element $f \in R$ given by (4) is right invariant if and only if

$$
\begin{aligned}
& c f=f c^{\alpha^{n}} \quad \text { for all } c \in K, \\
& t f=f\left(t+a_{1}-a_{1}^{\alpha}\right) .
\end{aligned}
$$

For we have seen that these conditions are necessary; conversely, when they hold, then by an easy induction on the degree, $g f=f g_{1}$ for any $g \in R$ and $f$ is then right invariant.

Given any endomorphism $\alpha$, an $\alpha$-derivation $\delta$ of $K$ and $n>0$, write $x^{\mu}=x^{\delta \alpha^{n}}-x^{\alpha^{n} \delta} ;$ then $\mu$ is clearly linear and

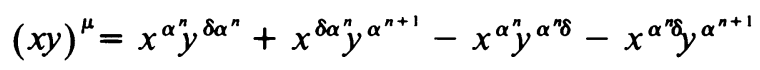

$$
\begin{aligned}
& =x^{\alpha^{n}} y^{\mu}+x^{\mu} y^{\alpha^{n+1}} \text {, }
\end{aligned}
$$

i.e. $\mu=\delta \alpha^{n}-\alpha^{n} \delta$ is an $\left(\alpha^{n}, \alpha^{n+1}\right)$-derivation. This derivation is necessarily inner when there is a right invariant element of degree $n$ :

Corollary. Let $R=K[t ; \alpha, \delta]$ be as in Theorem 3 and assume that $K$ has no inner automorphism which agrees with $\alpha$ on im $\alpha^{m}$ for any $m \geqslant 0$. If $f \in R$ of degree $n$, given by (4), is right invariant, then $\delta \alpha^{n}-\alpha^{n} \delta$ is inner. Moreover, 
if $\delta \alpha^{n}=\alpha^{n} \delta$, then $a_{1}^{\alpha}=a_{1}$ and $\alpha^{n}$ agrees with the inner automorphism of $K(t ; \alpha, \delta)$ induced by $f$, leaving $t$ fixed.

Proof. By the right invariance of $f$ we have for each $g \in R, g f=f g_{1}$ and it is clear that the mapping $g \mapsto g_{1}$ is an endomorphism of $R$. If we apply this endomorphism to (2) we obtain

$$
c^{\alpha^{n}}\left(t+a_{1}-a_{1}^{\alpha}\right)=\left(t+a_{1}-a_{1}^{\alpha}\right) c^{\alpha^{n+1}}+c^{\delta \alpha^{n}} .
$$

If we use (2) to simplify this relation, we obtain, on writing $a_{0}=a_{1}-a_{1}^{\alpha}$,

$$
c^{\alpha^{n}} a_{0}-a_{0} c^{\alpha^{n+1}}=c^{\delta \alpha^{n}}-c^{\alpha^{n \delta}} \text {. }
$$

This shows that $\mu=\delta \alpha^{n}-\alpha^{n} \delta$ is inner. If $\delta \alpha^{n}=\alpha^{n} \delta$ and $a_{0} \neq 0$, this means that the inner automorphism induced by $a_{0}$ agrees with $\alpha$ on im $\alpha^{n}$, a contradiction. Hence $a_{0}=0$ and now (6) reduces to $t f=f t$, thus the inner automorphism induced by $f$ leaves $t$ fixed and on $K$ agrees with $\alpha^{n}$.

In particular, if $\alpha$ and $\delta \alpha^{r}-\alpha^{r} \delta$ are outer for all $r>0$, this Corollary shows $R$ to be simple.

4. We can now describe the ideal structure of $K[t ; \alpha, \delta]$.

THEOREM 4. Let $R=K[t ; \alpha, \delta]$ be a skew polynomial ring, where $K$ has no inner automorphism which agrees with $\alpha^{r}$ on im $\alpha^{m}$ for any $r>0, m \geqslant 0$. Then the monic right invariant elements in $R$ form a monoid $S$, and either $S=\{1\}$ and $R$ is simple, or $S$ is generated by a single element $d \neq 1$ and the ideals of $R$ are all of the form $d^{\nu} R(\nu=0,1,2, \ldots)$.

Proof. We know that every ideal of $R$ is generated as a right ideal by a monic right invariant element, and if $f$ is monic right invariant of degree $n$, then by Theorem $3, c f=f c^{\alpha^{n}}$ for all $c \in K$. Given $f, g$ monic right invariant, the right ideal $f R+g R$ is two-sided, and hence of the form $f R+g R=d R$, where $d$ is again monic right invariant. Let us choose $d$ to be monic right invariant of least positive degree $r$; if $f$ is any monic right invariant of degree $n$, then by Theorem 3, $d^{n}$ and $f^{r}$ both induce $\alpha^{n r}$ on $K$; hence by Lemma 2 we have $f^{r}=d^{n} a$ for some $a \in K^{[\alpha]}$. By comparing leading terms we find that $a=1$, so

$$
f^{r}=d^{n}
$$

But if $f R+d R=h R$, then $h$ is again right invariant and of positive degree by (7); by the minimality of $r, h$ is associated to $d$ and it follows that $d R \supseteq f R$. Hence

$$
f=d^{\nu} g \text { for some } g \in R \text { and } \nu>0 .
$$

Now if $\operatorname{deg} f=n$, then $d f$ and $f d$ both induce $\alpha^{n+r}$ on $K$, hence as before (using Theorem 3 and Lemma 2) we find $f d=d f$. Together with (8) this shows that $g=d^{-\nu} f=f d^{-\nu}$, therefore $g$ induces $\alpha^{n-r \nu}$. By Theorem 3, tf $=f\left(t+a_{0}\right), t d^{\nu}=d^{\nu}\left(t+b_{0}\right)$ for some $a_{0}, b_{0} \in K$; hence

$$
\left(t+b_{0}\right) g=\left(t+b_{0}\right) d^{-v} f=d^{-\nu} t f=g\left(t+a_{0}\right) .
$$

By changing the variable from $t$ to $t+b_{0}$ we see that $g$ is again right 
invariant. Therefore if we choose $\nu$ in (8) maximal, we have $\operatorname{deg} g<r=$ $\operatorname{deg} d$, and by the minimality of $r$ we conclude that $g=1$ and $f=d^{\nu}$. This completes the proof.

If we combine this result with Theorem 1 we obtain

TheOREM 5. Let $R=K[t ; \alpha, \delta]$ be a skew polynomial ring, where $K$ has no inner automorphism agreeing with $\alpha^{r}$ on im $\alpha^{m}$ for any $r>0, m \geqslant 0$. Either (i) there is a nonconstant polynomial which is right invariant; if $d$ is monic right invariant of least positive degree, then $S=\left\{1, d, d^{2}, \ldots\right\}$ is a right denominator set and $R_{S}$ is a simple principal left and right ideal domain; or (ii) there is no nonconstant right invariant polynomial; then $R$ is a simple principal right ideal domain, which is left principal if and only if $\alpha$ is an automorphism of $K$.

To complete the proof in case (i) we observe that $R_{S}$ contains $K^{[\alpha]}$, because on $R_{S}, \alpha^{r}$ agrees with the inner automorphism induced by $d(r=\operatorname{deg} d)$. Now $R_{S}$ may be expressed as localization of $K^{[\alpha]}[t ; \alpha, \delta]$. Since $\alpha$ is an automorphism on $K^{[\alpha]}$, this is left, as well as right, principal and hence so is $R_{S}$. In case (ii) it is well known that $R$ is principal if and only if $\alpha$ is an automorphism.

For the special case $\delta=0$ this result has long been known (cf. [5] and, for an interesting application, [6]). A computational proof of (ii) (under rather stronger conditions) has been given by Cozzens [3] (cf. also [4]).

5. To construct an example where $R$ itself is simple (cf. [4, pp. 53-61]) we note that when $\alpha \delta=\delta \alpha$, a comparison of the coefficients of $t^{n-1}$ in (5) gives

$$
a_{1} c^{\alpha^{n}}-c^{\alpha^{n-1}} a_{1}=n c^{\alpha^{n-1} \delta} \text {. }
$$

Thus if char $K=0, \delta$ is an inner derivation on im $\alpha^{n-1}$, and to get a simple ring we need only choose $K$ so that $\alpha \delta=\delta \alpha$ and $\delta$ is not inner on im $\alpha^{m}$ for any $m \geqslant 0$. Of course we must also take $\alpha$ to be not an automorphism to get a ring which is not left principal.

Let $k$ be any commutative field of characteristic $0, X=\left\{x_{i j}\right\}, i, j=1$, $2, \ldots$, a family of noncommuting indeterminates and define

$$
x_{i j}^{\alpha}=x_{i+1 j}, \quad x_{i j}^{\delta}=x_{i j+1} .
$$

The free $k$-algebra $k\langle X\rangle$ has a universal field of fractions $K$ (cf. [2, Chapter 7]), $\alpha, \delta$ extend to $K$ and satisfy $\alpha \delta=\delta \alpha$; moreover, from the construction of $K$ it is clear that each element of $K$ involves only finitely many of the variables $x_{i j}$. Now if (9) were true, then

$$
x_{i j}=n^{-1}\left(a_{1} x_{i+1 j-1}-x_{i j-1} a_{1}\right) \quad \forall i>n, j>1 .
$$

We see that this is impossible by taking $i, j$ greater than the suffixes of all the variables occurring in $a_{1}$. Thus $\delta$ is not inner; that $\alpha$ is not an automorphism of $K$ is clear because $x_{11} \notin \operatorname{im} \alpha$.

\section{REFERENCES}

1. P. M. Cohn, Quadratic extensions of skew fields, Proc. London Math. Soc. (3) 11 (1961), 531-556. 
2. Free rings and their relations, LMS monographs, no. 2, Academic Press, London and New York, 1971.

3. J. H. Cozzens, Simple principal left ideal domains, J. Algebra 23 (1972), 66-75.

4. J. H. Cozzens and C. C. Faith, Simple Noetherian rings, Cambridge Tracts in Math., no. 69, Cambridge Univ. Press, 1975.

5. N. Jacobson, Theory of rings, Math. Surveys, vol. 1, Amer. Math. Soc., Providence, R. I., 1943.

6. B. L. Osofsky, On twisted polynomial rings, J. Algebra 18 (1971), 597-607.

Department of Mathematics, Bedford College, Regents Park, London NW1 4NS ENGLAND 